\title{
NONUNIQUENESS THEOREM FOR A SINGULAR CAUCHY-NICOLETTI PROBLEM
}

\author{
JOSEF KALAS
}

Received 20 September 2002

The problem of nonuniqueness for a singular Cauchy-Nicoletti boundary value problem is studied. The general nonuniqueness theorem ensuring the existence of two different solutions is given such that the estimating expressions are nonlinear, in general, and depend on suitable Lyapunov functions. The applicability of results is illustrated by several examples.

\section{Introduction}

The nonuniqueness of a regular or singular Cauchy problem for ordinary differential equations is studied in several papers such as $[3,4,5,13,14,15,16,17]$. Most of these results can also be found in the monograph [1]. The uniqueness of solutions of Cauchy initial value problem for ordinary differential equations with singularity is investigated in $[7,8,9,12]$. The topological structure of solution sets to a large class of boundary value problems for ordinary differential equations is studied in [2]. First results on the nonuniqueness for a singular Cauchy-Nicoletti boundary value problem are given in [10, 11,12 ] by Kiguradze, where sufficient conditions for the nonuniqueness are written in the form of one-sided inequalities for the components in the right-hand side $f\left(t, x_{1}, \ldots, x_{n}\right)$ of the corresponding equation. An expression for the estimation of the $j$ th component $f_{j}\left(t, x_{1}, \ldots, x_{n}\right)$ of $f$ depends on $t$ and $x_{j}$ and is linear in $\left|x_{j}\right|$.

In [6], we studied the nonuniqueness for a singular Cauchy problem. Our criteria involve vector Lyapunov functions and the estimations need not be linear. The present paper deals with the nonuniqueness of the singular Cauchy-Nicoletti problem and extends the results of [6] to this more general problem.

Supposing $-\infty \leq a<A \leq \infty, b>0$, we will use the following notations throughout the paper: $\mathbb{R}^{k}$ and $\mathbb{R}^{+}$denote $k$-dimensional real Euclidean space and the interval $[0, \infty)$, respectively. $|\cdot|$ is used for the notation of Hölder's 1-norm (the sum of the absolute values of components). $x=\left(x_{1}, \ldots, x_{n}\right)$ denotes a variable vector from $\mathbb{R}^{n}$ with components $x_{1}, \ldots, x_{n}$, while $x_{0}=\left(x_{01}, \ldots, x_{0 n}\right)$ stands for a fixed vector from $\mathbb{R}^{n}$ with components $x_{01}, \ldots, x_{0 n} . N$ is equal to the set $\{1, \ldots, n\} . l$ denotes a fixed number from the set $\{1, \ldots, n\}$. 
$i_{1}, i_{2}, \ldots, i_{l}$ are fixed integers such that $1 \leq i_{1}<i_{2}<\cdots<i_{l} \leq n . I$ is set to be equal to $\left\{i_{1}, \ldots, i_{l}\right\}$. Pr $x$ denotes a projection of $x$ such that $\operatorname{Pr} x=\left(x_{i_{1}}, \ldots, x_{i_{l}}\right)$, while $\operatorname{Pr}^{\star} x$ denotes a complementary projection to $\operatorname{Pr} x$. Clearly, $\operatorname{Pr}^{\star} x=\left(x_{j_{1}}, \ldots, x_{j_{n-l}}\right)$, where $1 \leq j_{1}<\cdots<$ $j_{n-l} \leq n,\left\{i_{1}, \ldots, i_{l}\right\} \cap\left\{j_{1}, \ldots, j_{n-l}\right\}=\varnothing . R_{\alpha, \beta ; b}^{k}\left(x_{0}\right)$ and $\tilde{R}_{a, A}^{k}$ are used for the notation of the set $\left\{(t, x) \in \mathbb{R}^{k+1}: \alpha<t<\beta,\left|x-x_{0}\right| \leq b\right\}$ and the set $\left\{(t, x) \in \mathbb{R}^{k+1}: a<t<A, x \in \mathbb{R}^{k}\right\}$, respectively. The symbol $\hat{R}_{a, A}^{n}$ will be used for the set $\left\{(t, x) \in \mathbb{R}^{n+1}: a \leq t \leq A, x \in \mathbb{R}^{n}\right\}$. $\Delta(\alpha, \beta)$ denotes the interval $(\min (\alpha, \beta), \max (\alpha, \beta))$.

The notation $C[\Gamma, \Omega]$ is used for the notation of the class of all continuous mappings $\Gamma \rightarrow \Omega . A C\left[[a, A], \mathbb{R}^{k}\right]$ and $\widetilde{A C}\left[[a, A], \mathbb{R}^{k}\right]$ denote the class of all absolutely continuous mappings $[a, A] \rightarrow \mathbb{R}^{k}$ and the class of all mappings from $C\left[[a, A], \mathbb{R}^{k}\right]$ which are absolutely continuous on any interval $[\alpha, \beta]$, where $a<\alpha<\beta<A$, respectively. The class of all Lebesgue-integrable mappings $[a, A] \rightarrow \mathbb{R}^{+}$is denoted by $L\left[[a, A], \mathbb{R}^{+}\right] . \mathscr{L}_{\tau}\left[\hat{R}_{a, A}^{n}, \mathbb{R}^{+k}\right]$ stands for the class of all functions $V(t, x): \hat{R}_{a, A}^{n} \rightarrow \mathbb{R}^{+k}$ with the following property: $V(t, \cdot)$ is uniformly continuous, and if $a<\alpha<\beta<A, \tau \notin[\alpha, \beta]$, then $V(t, x(t))$ is absolutely continuous on $[\alpha, \beta]$ for any absolutely continuous function $x:[\alpha, \beta] \rightarrow \mathbb{R}^{n}$. $K_{\sigma_{1}, \ldots, \sigma_{p}}\left[\hat{R}_{a, A}^{k}, \mathbb{R}^{m}\right]$ denotes the class of all mappings $\hat{R}_{a, A}^{k} \rightarrow \mathbb{R}^{m}$ which satisfy Carathéodory conditions on $R_{\alpha, \beta ; \varrho}^{k}(0)$ for any $\alpha, \beta, a \leq \alpha<\beta \leq A, \sigma_{j} \notin[\alpha, \beta](j=1, \ldots, p), \varrho \in(0, \infty)$, $\sigma_{1}, \ldots, \sigma_{p}$ being numbers from $[a, A] . N_{0}\left(a, A ; \tau_{1}, \ldots, \tau_{n}\right)$ is used for the notation of the class $\left\{\Lambda=\left(\lambda_{i j}(t)\right)_{i, j=1}^{n}: \lambda_{i j} \in L\left[[a, A], \mathbb{R}^{+}\right]\right\}$such that the system of differential inequalities $\left|x_{i}^{\prime}(t)\right| \leq \sum_{j=1}^{n} \lambda_{i j}(t)\left|x_{j}(t)\right|, t \in[a, A], i \in N$, possesses no nontrivial solution $x(t)=$ $\left(x_{1}(t), \ldots, x_{n}(t)\right) \in A C\left[[a, A], \mathbb{R}^{n}\right]$ satisfying $x_{i}\left(\tau_{i}\right)=0(i=1, \ldots, n)$.

The fundamental role in the proof of our main theorem will be played by the following theorem by Kiguradze, which is adapted from [12] (see also [10]) in a simplified form.

Kiguradze Theorem . Let $a \leq \tau_{i} \leq A, \hat{x}_{0 i} \in \mathbb{R}$ for $i=1, \ldots, n$. Suppose that $f \in K_{\sigma_{1}, \ldots, \sigma_{p}}\left[\hat{R}_{a, A}^{n}\right.$, $\mathbb{R}^{n}$. Assume that the components $f_{i}$ of $f$ satisfy

$$
f_{i}(t, x) \operatorname{sgn}\left[\left(t-\tau_{i}\right)\left(x_{i}-\hat{x}_{0 i}\right)\right] \leq \sum_{j=1}^{n} \lambda_{i j}(t)\left|x_{j}\right|+\mu_{i}(t) \quad(i=1, \ldots, n)
$$

for $(t, x)=\left(t, x_{1}, \ldots, x_{n}\right) \in \tilde{R}_{a, A}^{n}$, where $\hat{x}_{0 i}=0$ if $\tau_{i} \in\left\{\sigma_{1}, \ldots, \sigma_{p}\right\}$. Suppose that $\Lambda(t)=$ $\left(\lambda_{i j}(t)\right)_{i, j=1}^{n} \in N_{0}\left(a, A ; \tau_{1}, \ldots, \tau_{n}\right), \mu_{i} \in L\left[[a, A], \mathbb{R}^{+}\right]$. Then the Cauchy-Nicoletti problem

$$
x^{\prime}=f(t, x), \quad x_{i}\left(\tau_{i}\right)=0 \quad(i=1, \ldots, n)
$$

has at least one solution $x(t)=\left(x_{1}(t), \ldots, x_{n}(t)\right) \in A C\left[[a, A], \mathbb{R}^{n}\right]$.

\section{Results}

Consider a Cauchy-Nicoletti boundary value problem

$$
x^{\prime}=f(t, x), \quad x_{i}\left(t_{i}\right)=x_{0 i} \quad(i=1, \ldots, n),
$$

where $f(t, x)=\left(f_{1}\left(t, x_{1}, \ldots, x_{n}\right), \ldots, f_{n}\left(t, x_{1}, \ldots, x_{n}\right)\right), f \in K_{\sigma_{1}, \ldots, \sigma_{p}}\left[\hat{R}_{a, A}^{n}, \mathbb{R}^{n}\right], x_{0 i} \in \mathbb{R}$, and $t_{i} \in[a, A](i \in N)$. 
Theorem 2.1. Suppose that there are numbers $c_{i} \in \mathbb{R}(i \in N), B_{i} \in[a, A] \backslash\left\{t_{i}, \sigma_{1}, \ldots, \sigma_{p}\right\}$ $(i \in I)$, a matrix function $\Lambda=\left(\lambda_{i j}\right)_{i, j=1}^{n} \in N_{0}\left(a, A ; \tau_{1}, \ldots, \tau_{n}\right)$ and functions $\mu_{i} \in L\left[[a, A], \mathbb{R}^{+}\right]$ $(i \in N)$ such that $c_{i}=x_{0 i}$ for $i \in N \backslash I$ and

$$
f_{i}(t, x)\left[\operatorname{sgn}\left(t-\tau_{i}\right)\left(x_{i}-c_{i}\right)\right] \leq \sum_{j=1}^{n} \lambda_{i j}(t)\left|x_{j}\right|+\mu_{i}(t) \quad(i \in N)
$$

holds for $(t, x)=\left(t, x_{1}, \ldots, x_{n}\right) \in \tilde{\mathbb{R}}_{a, A}^{n}$, where $\tau_{i}=t_{i}$ or $\tau_{i}=B_{i}$ whenever $i \in N \backslash I$ or $i \in I$, respectively.

Assume that

(i) there exist vector functions $g_{i}=\left(g_{i 1}, \ldots, g_{i k_{i}}\right) \in K_{a, A, t_{i}, B_{i}}\left[\hat{\mathbb{R}}_{a, A}^{k_{i}}, \mathbb{R}^{k_{i}}\right](i \in I)$ such that $\operatorname{sgn}\left(t-t_{i}\right) g_{i j}\left(t, u_{1}, \ldots, u_{j-1}, \cdot, u_{j}, \ldots, u_{k_{i}}\right)$ is nondecreasing for $j=1, \ldots, k_{i}$ and there is a solution $\varphi_{i}(t)=\left(\varphi_{i 1}(t), \ldots, \varphi_{i k_{i}}(t)\right)$ of

$$
u_{i}^{\prime}=g_{i}\left(t, u_{1}, \ldots, u_{k_{i}}\right)
$$

satisfying

$$
\varphi_{i}(t)>0 \quad \text { for } t \in \Delta\left(t_{i}, B_{i}\right), \quad \lim _{t \rightarrow t_{i}} \varphi_{i}(t)=0, \quad \liminf _{t \rightarrow B_{i}} \varphi_{i}(t)>0
$$

for $i \in I$;

(ii) $V_{i}(t, x)=\left(V_{i 1}(t, x), \ldots, V_{i k_{i}}(t, x)\right) \in \mathscr{L}_{t_{i}}\left[\hat{R}_{a, A}^{n}, \mathbb{R}^{+k_{i}}\right](i \in I)$ are such that there exists $y_{0} \in \mathbb{R}^{l}$ with the property

$$
\begin{gathered}
\sup \left\{V_{i j}\left(B_{i}, y\right): y \in \mathbb{R}^{n}, \operatorname{Pr} y=y_{0}\right\}<\liminf _{t \rightarrow B_{i}} \varphi_{i j}(t) \quad\left(j=1, \ldots, k_{i}\right) \\
\left|V_{i}(t, x)\right| \geq \Psi_{i}\left(\left|x_{i}-z_{i}(t)\right|\right) \quad \text { for } t \in \Delta\left(t_{i}, B_{i}\right),
\end{gathered}
$$

where $\Psi_{i} \in C\left[\mathbb{R}^{+}, \mathbb{R}^{+}\right], z_{i} \in C[(a, A), \mathbb{R}]$ are such that

$$
\Psi_{i}(0)=0, \quad \Psi_{i}(u)>0 \quad \text { for } u>0, \quad \lim _{t \rightarrow t_{i}} z_{i}(t)=x_{0 i}
$$

for $i \in I$;

(iii) there exist positive functions $\varepsilon_{i k} \in C\left[(a, A), \mathbb{R}^{+}\right]\left(i \in I ; k=1, \ldots, k_{i}\right)$ such that

$$
\begin{aligned}
& \operatorname{sgn}\left(B_{i}-t_{i}\right) V_{i j}^{\prime}(t, x(t)) \\
& \quad \geq \operatorname{sgn}\left(B_{i}-t_{i}\right) g_{i j}\left(t, \varphi_{i 1}(t), \ldots, \varphi_{i, j-1}(t), V_{i j}(t, x(t)), \varphi_{i, j+1}(t), \ldots, \varphi_{i k_{i}}(t)\right)
\end{aligned}
$$

holds for $i \in I, j=1, \ldots, k_{i}$, and for any solution $x(t)$ of (2.1) a.e. on any interval $\left(\alpha_{i 1}, \alpha_{i 2}\right) \subseteq \Delta\left(t_{i}, B_{i}\right)$ for which

$$
\begin{gathered}
V_{i k}(t, x(t))<\varphi_{i k}(t)+\varepsilon_{i k}(t) \quad \text { on }\left(\alpha_{i 1}, \alpha_{i 2}\right)\left(k=1, \ldots, k_{i}\right), \\
V_{i j}(t, x(t))>\varphi_{i j}(t) \quad \text { on }\left(\alpha_{i 1}, \alpha_{i 2}\right) .
\end{gathered}
$$

Then the Cauchy-Nicoletti boundary value problem (2.1) has at least two different solutions on $[a, A]$, either of which satisfies $V_{i}(t, x(t)) \leq \varphi_{i}(t)$ for $t \in \Delta\left(t_{i}, B_{i}\right)$ and $i \in I$. 
594 Nonuniqueness for a singular Cauchy-Nicoletti problem

Proof. Without loss of generality, it can be assumed that $I=\{1, \ldots, l\}$,

$$
\operatorname{Pr} x=\left(x_{1}, \ldots, x_{l}\right), \quad \operatorname{Pr}^{\star} x=\left(x_{l+1}, \ldots, x_{n}\right) .
$$

For any $i \in I$ and $j \in\left\{1, \ldots, k_{i}\right\}$, denote

$$
L_{i j}=\liminf _{t \rightarrow B_{i}} \varphi_{i j}(t), \quad S_{i j}=\sup \left\{V_{i j}\left(B_{i}, y\right): y \in \mathbb{R}^{n}, \operatorname{Pr} y=y_{0}\right\}
$$

According to (2.5) and to the uniform continuity of $V_{i j}\left(B_{i}, \cdot\right)$, we have a relation

$$
\begin{aligned}
V_{i j}\left(B_{i}, y^{*}\right) & \leq V_{i j}\left(B_{i}, y\right)+V_{i j}\left(B_{i}, y^{*}\right)-V_{i j}\left(B_{i}, y\right) \\
& \leq \frac{1}{2}\left(L_{i j}+S_{i j}\right)+\frac{1}{4}\left(L_{i j}-S_{i j}\right)=\frac{3}{4} L_{i j}+\frac{1}{4} S_{i j}<L_{i j}
\end{aligned}
$$

for $y \in \mathbb{R}^{n}$, Pr $y=y_{0}$, and for $y^{*} \in \mathbb{R}^{n}$ sufficiently close to $y$. Hence it can be supposed without loss of generality that $y_{0} \neq \operatorname{Pr} x_{0}$.

Further, the uniform continuity of $V_{i j}\left(B_{i}, \cdot\right)$ implies that the inequality

$$
\sup \left\{V_{i j}\left(B_{i}, y\right): y \in \mathbb{R}^{n}, \operatorname{Pr} y=y_{0}-\lambda\left(y_{0}-\operatorname{Pr} x_{0}\right)\right\}<\liminf _{t \rightarrow B_{i}} \varphi_{i j}(t) \quad\left(i \in I ; j=1, \ldots, k_{i}\right)
$$

holds provided that $\lambda>0$ is sufficiently small. Therefore, we can choose $\tilde{x}_{1}, \tilde{x}_{2} \in \mathbb{R}^{l}, \tilde{x}_{1} \neq$ $\tilde{x}_{2}$, such that

$$
\max _{k=1,2}\left[\sup \left\{V_{i j}\left(B_{i}, y\right): y \in \mathbb{R}^{n}, \operatorname{Pr} y=\tilde{x}_{k}\right\}\right]<\liminf _{t \rightarrow B_{i}} \varphi_{i j}(t) \quad\left(i=1, \ldots, l ; j=1, \ldots, k_{i}\right) .
$$

Choose $\tilde{\xi} \in\left\{\tilde{x}_{1}, \tilde{x}_{2}\right\}$ arbitrary. Put $\xi=x_{0}-\left(\tilde{\xi}, \operatorname{Pr}^{\star} x_{0}\right), X=x-x_{0}+\xi$, and $f^{*}(t, X)=$ $f\left(t, x_{0}+X-\xi\right)$ for $(t, X)=\left(t, X_{1}, \ldots, X_{n}\right) \in \hat{R}_{a, A}^{n}$.

Clearly $f^{*} \in K_{\sigma_{1}, \ldots, \sigma_{p}}\left[\hat{R}_{a, A}^{n}, \mathbb{R}^{n}\right]$. By using (2.2), we obtain

$$
\begin{aligned}
f_{i}^{*}(t, X) \operatorname{sgn}\left[\left(t-\tau_{i}\right)\left(X_{i}+\tilde{\xi}_{i}-c_{i}\right)\right] & \leq \sum_{j=1}^{l} \lambda_{i j}(t)\left|X_{j}+\tilde{\xi}_{j}\right|+\sum_{j=l+1}^{n} \lambda_{i j}(t)\left|X_{j}+x_{0 j}\right|+\mu_{i}(t) \\
& \leq \sum_{j=1}^{n} \lambda_{i j}(t)\left|X_{j}\right|+\tilde{\mu}_{i}(t)
\end{aligned}
$$

for $(t, X) \in \tilde{R}_{a, A}^{n}, i=1, \ldots, l$, and

$$
\begin{aligned}
f_{i}^{*}(t, X) \operatorname{sgn}\left[\left(t-\tau_{i}\right) X_{i}\right] & \leq \sum_{j=1}^{l} \lambda_{i j}(t)\left|X_{j}+\tilde{\xi}_{j}\right|+\sum_{j=l+1}^{n} \lambda_{i j}(t)\left|X_{j}+x_{0 j}\right|+\mu_{i}(t) \\
& \leq \sum_{j=1}^{n} \lambda_{i j}(t)\left|X_{j}\right|+\tilde{\mu}_{i}(t)
\end{aligned}
$$


for $(t, X) \in \tilde{R}_{a, A}^{n}, i=l+1, \ldots, n$, where

$$
\tilde{\mu}_{i}(t)=\sum_{j=1}^{l} \lambda_{i j}(t)\left|\tilde{\xi}_{j}\right|+\sum_{j=l+1}^{n} \lambda_{i j}(t)\left|x_{0 j}\right|+\mu_{i}(t)
$$

for $i=1,2, \ldots, n$. As $\tilde{\mu}_{i} \in L\left[[a, A], \mathbb{R}^{+}\right]$holds, Kiguradze theorem implies that the boundary value problem

$$
X^{\prime}=f^{*}(t, X), \quad X_{i}\left(\tau_{i}\right)=0 \quad(i=1, \ldots, n)
$$

has at least one solution $X(t) \in A C\left[[a, A], \mathbb{R}^{n}\right]$. Hence $x(t)=X(t)+x_{0}-\xi$ is a solution of

$$
\begin{gathered}
x^{\prime}=f(t, x), \quad x_{i}\left(\tau_{i}\right)=\tilde{\xi}_{i} \quad(i=1, \ldots, l), \\
x_{i}\left(\tau_{i}\right)=x_{0 i} \quad(i=l+1, \ldots, n) .
\end{gathered}
$$

Now we will prove that $\lim _{t \rightarrow t_{i}} x_{i}(t)=x_{0 i}$ for $i=1, \ldots, l$. Put $m_{i}(t)=V_{i}(t, x(t)), m_{i j}(t)=$ $V_{i j}(t, x(t))$ for $i=1, \ldots, l$ and $j=1, \ldots, k_{i}$. In view of (2.14), the inequality

$$
m_{i}(t)<\varphi_{i}(t)
$$

holds for $t \in(a, A)$ sufficiently close to $B_{i}$. Suppose for definiteness that $t_{i}<B_{i}$, that is, $\Delta\left(t_{i}, B_{i}\right)=\left(t_{i}, B_{i}\right)$ for some $i \in\{1, \ldots, l\}$. We will show that $m_{i}(t) \leq \varphi_{i}(t)$ for $t \in\left(t_{i}, B_{i}\right)$. Assume on the contrary that there is a $\tau \in\left(t_{i}, B_{i}\right)$ such that $m_{i}(\tau) \leq \varphi_{i}(\tau)$ is not true. Since $x(t)$ is continuous and (2.20) holds for $t \in(a, A)$ sufficiently close to $B_{i}$, there exist $j \in\left\{1, \ldots, k_{i}\right\}$ and an interval $J_{i}=\left(\tau_{i 1}, \tau_{i 2}\right)$ such that $\tau<\tau_{i 1}<\tau_{i 2}<B_{i}$,

$$
\begin{gathered}
m_{i j}\left(\tau_{i 2}\right)=\varphi_{i j}\left(\tau_{i 2}\right), \\
\varphi_{i j}(s)<m_{i j}(s)<\varphi_{i j}(s)+\varepsilon_{i j}(s) \quad \text { for } s \in J_{i}, \\
m_{i k}(s)<\varphi_{i k}(s)+\varepsilon_{i k}(s) \quad \text { for } s \in J_{i}, k=1, \ldots, k_{i} .
\end{gathered}
$$

Using (2.8), we get

$$
m_{i j}^{\prime}(s) \geq g_{i j}\left(s, \varphi_{i 1}(s), \ldots, \varphi_{i, j-1}(s), m_{i j}(s), \varphi_{i, j+1}(s), \ldots, \varphi_{i k_{i}}(s)\right)
$$

a.e. on $J_{i}$. As $g_{i j}\left(t, u_{1}, \ldots, u_{j-1}, \cdot, u_{j+1}, \ldots, u_{n}(s)\right)$ is nondecreasing, we have

$$
m_{i j}^{\prime}(s) \geq g_{i j}\left(s, \varphi_{i 1}(s), \ldots, \varphi_{i k_{i}}(s)\right)=\varphi_{i j}^{\prime}(s)
$$

a.e. on $J_{i}$. Therefore, the function $m_{i j}(t)-\varphi_{i j}(t)$ is nondecreasing on $J_{i}$, which is a contradiction to $m_{i j}\left(\tau_{i 2}\right)=\varphi_{i j}\left(\tau_{i 2}\right)$. Thus

$$
0 \leq m_{i}(t) \leq \varphi_{i}(t) \quad \text { for } t \in\left(t_{i}, B_{i}\right) .
$$

Now the condition $\lim _{t \rightarrow t_{i}+} \varphi_{i}(t)=0$ implies $\lim _{t \rightarrow t_{i}+} m_{i}(t)=0$. With respect to the continuity of $x_{i}(t)$ on $[a, A]$, we have $x_{i}\left(t_{i}\right)=\lim _{t \rightarrow t_{i}} x_{i}(t)=x_{0 i}$. The inequality (2.24) implies $V_{i}(t, x(t)) \leq \varphi_{i}(t)$ for $t \in \Delta\left(t_{i}, B_{i}\right)$. 
Corollary 2.2. Let $c_{i} \in \mathbb{R}(i \in N), B_{i} \in[a, A] \backslash\left\{t_{i}, \sigma_{1}, \ldots, \sigma_{p}\right\}$ ( $\left.i \in I\right)$, a matrix function $\Lambda=\left(\lambda_{i j}\right)_{i, j=1}^{n} \in N_{0}\left(a, A ; \tau_{1}, \ldots, \tau_{n}\right)$, and functions $\mu_{i} \in L\left[[a, A], \mathbb{R}^{+}\right](i \in N)$ be such that $c_{i}=x_{0 i}$ for $i \in N \backslash I$ and condition (2.2) is fulfilled, where $\tau_{i}=t_{i}$ or $\tau_{i}=B_{i}$ whenever $i \in$ $N \backslash I$ or $i \in I$, respectively.

Assume that

(i) there exist functions $g_{i} \in K_{a, A, t_{i}, B_{i}}\left[\hat{R}_{a, A}^{1}, \mathbb{R}\right](i \in I)$ such that $\operatorname{sgn}\left(t-t_{i}\right) g_{i}(t, \cdot)$ are nondecreasing and there are solutions $\varphi_{i}(t)$ of

$$
u_{i}^{\prime}=g_{i}\left(t, u_{i}\right)
$$

satisfying (2.4);

(ii) there are $z_{i} \in \widetilde{A C}[[a, A], \mathbb{R}]$ and $\varepsilon=\left(\varepsilon_{i_{1}}, \ldots, \varepsilon_{i_{l}}\right) \in C\left[(a, A), \mathbb{R}^{+l}\right]$ such that $z_{i}\left(t_{i}\right)=$ $x_{0 i}(i \in I)$ and the estimation

$$
\operatorname{sgn}\left(B_{i}-t_{i}\right) \operatorname{sgn}\left(x_{i}-z_{i}(t)\right)\left(f_{i}(t, x)-z_{i}^{\prime}(t)\right) \geq \operatorname{sgn}\left(B_{i}-t_{i}\right) g_{i}\left(t,\left|x_{i}-z_{i}(t)\right|\right)(i \in I)
$$

is fulfilled on $\hat{\Omega}=\left\{(t, x): \varphi_{i}(t)<\left|x_{i}-z_{i}(t)\right|<\varphi_{i}(t)+\varepsilon_{i}(t), t \in \Delta\left(t_{i}, B_{i}\right)\right\}$ for almost all $t \in \Delta\left(t_{i}, B_{i}\right)$. Then the Cauchy-Nicoletti boundary value problem (2.1) has at least two different solutions on $[a, A]$, either of which satisfies $\left|x_{i}(t)-z_{i}(t)\right| \leq \varphi_{i}(t)$ for $t \in \Delta\left(t_{i}, B_{i}\right)$ and $i \in I$.

Proof. Without loss of generality, it can be supposed that $I=\{1, \ldots, l\}$ and $\operatorname{Pr} x=\left(x_{1}, \ldots\right.$, $\left.x_{l}\right)$. Put $k_{i}=1$ and $V_{i}(t, x(t))=V_{i 1}(t, x)=\left|x_{i}-z_{i}(t)\right|$ for $i=1, \ldots, l$. Then

$$
\begin{aligned}
\operatorname{sgn}\left(B_{i}-t_{i}\right) V_{i 1}^{\prime}(t, x(t)) & \geq \operatorname{sgn}\left(B_{i}-t_{i}\right)\left(f_{i}(t, x(t))-z_{i}^{\prime}(t)\right) \operatorname{sgn}\left(x_{i}(t)-z_{i}(t)\right) \\
& \geq \operatorname{sgn}\left(B_{i}-t_{i}\right) g_{i}\left(t,\left|x_{i}(t)-z_{i}(t)\right|\right) \\
& =\operatorname{sgn}\left(B_{i}-t_{i}\right) g_{i}\left(t, V_{i 1}(t, x(t))\right)
\end{aligned}
$$

holds for any solution $x(t)$ of (2.1) a. e. on any interval $\left(\alpha_{i 1}, \alpha_{i 2}\right) \subseteq \Delta\left(t_{i}, B_{i}\right)$ for which $\varphi_{i}(t)<V_{i}(t, x(t))<\varphi_{i}(t)+\varepsilon_{i}(t)$ on $\left(\alpha_{i 1}, \alpha_{i 2}\right)$. The assumptions of Theorem 2.1 are satisfied.

Example 2.3. Let $f_{1}, \ldots, f_{n} \in K_{0}\left[\hat{R}_{0,1}^{n}, \mathbb{R}\right]$ be such that

$$
\begin{gathered}
f_{1}\left(t, x_{1}, \ldots, x_{n}\right) \operatorname{sgn} x_{1} \geq \delta(t)\left|x_{1}\right|^{\gamma} \\
-f_{j}\left(t, x_{1}, \ldots, x_{n}\right) \operatorname{sgn} x_{j} \leq \sum_{k=1}^{j} \lambda_{j k}(t)\left|x_{k}\right|+\mu_{j}(t) \quad(j=2, \ldots, n)
\end{gathered}
$$

for $\left(t, x_{1}, \ldots, x_{n}\right) \in \tilde{R}_{0,1}^{n}$, where $\gamma \in(0,1)$ and $\delta, \lambda_{j k}, \mu_{j} \in L\left[[0,1], \mathbb{R}^{+}\right], \delta$ being a positive function. Consider the boundary value problem

$$
\begin{array}{cl}
x_{1}^{\prime}=f_{1}\left(t, x_{1}, \ldots, x_{n}\right), & x_{1}(0)=0, \\
x_{2}^{\prime}=f_{2}\left(t, x_{1}, \ldots, x_{n}\right), & x_{2}(1)=0, \\
\vdots & \\
x_{n}^{\prime}=f_{n}\left(t, x_{1}, \ldots, x_{n}\right), & x_{n}(1)=0 .
\end{array}
$$


Put $t_{1}=0, t_{2}=t_{3}=\cdots=t_{n}=1$,

$$
g_{1}(t, u)=\left\{\begin{array}{l}
\delta(t) u^{\gamma} \quad \text { for } u \geq 0 \\
0 \quad \text { for } u<0
\end{array}\right.
$$

$\lambda_{1 k}(t) \equiv 0(k=1, \ldots, n), \lambda_{j k}(t) \equiv 0(j=2, \ldots, n ; k=j+1, \ldots, n)$, and $\mu_{1}(t) \equiv 0$. Let $B_{1}=1$. Then $\tau_{1}=\tau_{2}=\cdots=\tau_{n}=1$,

$$
\begin{gathered}
f_{1}\left(t, x_{1}, \ldots, x_{n}\right) \operatorname{sgn}\left[\left(t-B_{1}\right) x_{1}\right] \leq 0, \\
f_{j}\left(t, x_{1}, \ldots, x_{n}\right) \operatorname{sgn}\left[(t-1) x_{j}\right] \leq \sum_{k=1}^{n} \lambda_{j k}(t)\left|x_{k}\right|+\mu_{j}(t) \quad(j=2, \ldots, n),
\end{gathered}
$$

and the equation $u_{1}^{\prime}=g_{1}(t, u)$ has a positive solution

$$
\varphi_{1}(t)=\left[(1-\gamma) \int_{0}^{t} \delta(s) d s\right]^{1 /(1-\gamma)}
$$

in $(0,1]$ such that $\lim _{t \rightarrow 0} \varphi_{1}(t)=0$. The assumptions of Corollary 2.2 are fulfilled with $I=\{1\}, c_{1}=0$, and $z(t)=z_{1}(t) \equiv 0$. Therefore, the considered boundary value problem has at least two different solutions on $[a, A]$. Moreover, the first component $x_{1}(t)$ of these solutions satisfies $\left|x_{1}(t)\right| \leq \varphi_{1}(t)$ for $t \in(0,1]$.

Corollary 2.4. Suppose that $-\infty<a<A<\infty, c \in \mathbb{R}, \lambda \in L\left[[a, A], \mathbb{R}^{+}\right]$, and $\mu \in L[[a, A]$, $\left.\mathbb{R}^{+}\right]$. Let $B \in[a, A] \backslash\left\{t_{n}, \sigma_{1}, \ldots, \sigma_{p}\right\}$ be such that

$$
\tilde{f}\left(t, x_{1}, \ldots, x_{n}\right) \operatorname{sgn}\left[(t-B)\left(x_{n}-c\right)\right] \leq \lambda(t)\left|x_{n}\right|+\mu(t)
$$

for $(t, x) \in \tilde{R}_{a, A}^{n}$. Assume that

(i) there exists a function $q \in K_{a, A, t_{n}, B}\left[\hat{R}_{a, A}^{1}, \mathbb{R}\right]$ such that $\operatorname{sgn}\left(t-t_{n}\right) q(t, \cdot)$ is nondecreasing and there is a solution $\varphi(t)$ of

$$
u^{\prime}=q(t, u)
$$

satisfying

$$
\varphi(t)>0 \quad \text { for } t \in \Delta\left(t_{n}, B\right), \quad \lim _{t \rightarrow t_{n}} \varphi(t)=0, \quad \liminf _{t \rightarrow B} \varphi(t)>0
$$

(ii) there are $z \in \widetilde{A C}[[a, A], \mathbb{R}]$ and $\varepsilon \in C\left[(a, A), \mathbb{R}^{+}\right]$such that $z\left(t_{n}\right)=x_{0 n}$ and

$$
\operatorname{sgn}\left(B-t_{n}\right) \operatorname{sgn}\left(x_{n}-z(t)\right)\left(\tilde{f}\left(t, x_{1}, \ldots, x_{n}\right)-z^{\prime}(t)\right) \geq \operatorname{sgn}\left(B-t_{n}\right) q\left(t,\left|x_{n}-z(t)\right|\right)
$$


598 Nonuniqueness for a singular Cauchy-Nicoletti problem

holds on $\hat{\Omega}=\left\{\left(t, x_{1}, \ldots, x_{n}\right): \varphi(t)<\left|x_{n}-z(t)\right|<\varphi(t)+\varepsilon(t), t \in \Delta\left(t_{n}, B\right)\right\}$ for almost all $t \in \Delta\left(t_{n}, B\right)$. Then the boundary value problem

$$
\begin{gathered}
v^{(n)}=\tilde{f}\left(t, v, v^{\prime}, \ldots, v^{(n-1)}\right), \\
v\left(t_{1}\right)=x_{01}, \quad v^{\prime}\left(t_{2}\right)=x_{02}, \ldots, \quad v^{(n-1)}\left(t_{n}\right)=x_{0 n}
\end{gathered}
$$

has at least two different solutions on $[a, A]$.

Proof. Put $I=\{n\}, k_{1}=1, \operatorname{Pr} x=x_{n}, c_{n}=c, g_{n}(t, u)=q(t, u), \varphi_{n}(t)=\varphi(t), c_{i}=x_{0 i}$ for $i=1, \ldots, n-1, \mu_{i}(t)=0$ for $i=1, \ldots, n-1, \mu_{n}(t)=\mu(t), B_{n}=B$, and

$$
\lambda_{i j}(t)= \begin{cases}1 & \text { for } 1 \leq i=j-1 \leq n-1 \\ \lambda(t) & \text { for } i=j=n \\ 0 & \text { otherwise. }\end{cases}
$$

Considering the system

$$
\begin{aligned}
& x_{1}^{\prime}=x_{2}, \\
& x_{1}\left(t_{1}\right)=x_{01}, \\
& x_{2}^{\prime}=x_{3} \text {, } \\
& x_{2}\left(t_{2}\right)=x_{02} \text {, } \\
& \vdots \\
& x_{n-1}^{\prime}=x_{n}, \\
& x_{n-1}\left(t_{n-1}\right)=x_{0 n-1}, \\
& x_{n}^{\prime}=\tilde{f}\left(t, x_{1}, x_{2}, \ldots, x_{n}\right), \\
& x_{n}\left(t_{n}\right)=x_{0 n},
\end{aligned}
$$

and applying Corollary 2.2, we get

$$
\begin{aligned}
f_{n}\left(t, x_{1}, \ldots, x_{n}\right) \operatorname{sgn}\left[\left(t-B_{n}\right)\left(x_{n}-c_{n}\right)\right] & \leq \sum_{j=1}^{n} \lambda_{n j}(t)\left|x_{j}\right|+\mu_{n}(t), \\
f_{i}\left(t, x_{1}, \ldots, x_{n}\right) \operatorname{sgn}\left[\left(t-t_{i}\right)\left(x_{i}-c_{i}\right)\right] \leq\left|x_{i+1}\right| & \leq \lambda_{i, i+1}\left|x_{i+1}\right| \\
& =\sum_{j=1}^{n} \lambda_{i j}(t)\left|x_{j}\right|+\mu_{i}(t)
\end{aligned}
$$

for $i=1, \ldots, n-1$. The result follows from Corollary 2.2.

Example 2.5. Let $\gamma \in(0,1)$. Consider the boundary value problem

$$
v^{\prime \prime}=p_{1}(t, v)\left|v^{\prime}\right|^{\gamma} \operatorname{sgn} v^{\prime}+p_{2}\left(t, v, v^{\prime}\right), \quad v(0)=0, \quad v^{\prime}(1)=0,
$$

where $p_{1} \in K_{1}\left[\hat{R}_{0,1}^{1}, \mathbb{R}\right]$ and $p_{2} \in K_{1}\left[\hat{R}_{0,1}^{2}, \mathbb{R}\right]$ are such that

$$
\begin{gathered}
x_{2} p_{2}\left(t, x_{1}, x_{2}\right) \leq 0 \quad \text { for }\left(t, x_{1}, x_{2}\right) \in(0,1) \times \mathbb{R}^{2}, \\
p_{1}\left(t, x_{1}\right) \leq-\delta(t) \quad \text { for }\left(t, x_{1}\right) \in(0,1) \times \mathbb{R},
\end{gathered}
$$

$\delta \in L[[0,1], \mathbb{R}]$ being a positive function. Since

$$
-p_{1}\left(t, x_{1}\right)\left|x_{2}\right|^{\gamma}-p_{2}\left(t, x_{1}, x_{2}\right) \operatorname{sgn} x_{2} \geq \delta(t)\left|x_{2}\right|^{\gamma},
$$


the assumptions of Corollary 2.4 are fulfilled with $n=2, a=0, A=1, t_{1}=0, t_{2}=1, c=0$, $B=0, z(t) \equiv 0, \lambda(t) \equiv 0, \mu(t) \equiv 0$, and

$$
q(t, u)=\left\{\begin{array}{ll}
-\delta(t) u^{\gamma} & \text { for } u \geq 0, \\
0 & \text { for } u<0,
\end{array} \quad \varphi(t)=\left[(1-\gamma) \int_{t}^{1} \delta(s) d s\right]^{1 / 1-\gamma}\right.
$$

Therefore, problem (2.41) has at least two different solutions on $[0,1]$.

Corollary 2.6. Let the assumptions of Corollary 2.2 be fulfilled with the exception that the conditions (i), (ii) are replaced by $\left(i^{\prime}\right),\left(i i^{\prime}\right)$ :

(i') there exist functions $h_{i}, q_{i} \in K_{a, A, t_{i}, B_{i}}\left[\hat{R}_{a, A}^{1}, \mathbb{R}\right](i \in I)$ such that functions $\operatorname{sgn}(t-$ $\left.t_{i}\right) h_{i}(t, \cdot)$ and $\operatorname{sgn}\left(t-t_{i}\right) q_{i}(t, \cdot)$ are nondecreasing for $i \in I$ and there are solutions $\varphi_{i}(t), \psi_{i}(t)$ of $u_{i}^{\prime}=h_{i}\left(t, u_{i}\right)$ and $v_{i}^{\prime}=q_{i}\left(t, v_{i}\right)$, respectively, satisfying

$$
\begin{array}{llll}
\varphi_{i}(t)>0 & \text { for } t \in \Delta\left(t_{i}, B_{i}\right), & \lim _{t \rightarrow t_{i}} \varphi(t)=0, & \liminf _{t \rightarrow B_{i}} \varphi(t)>0, \\
\psi_{i}(t)>0 & \text { for } t \in \Delta\left(t_{i}, B_{i}\right), & \lim _{t \rightarrow t_{i}} \psi(t)=0, & \liminf _{t \rightarrow B_{i}} \psi(t)>0
\end{array}
$$

for $t \in I$;

(ii') there are $z_{i} \in \widetilde{A C}[[a, A], \mathbb{R}]$ and $\varepsilon=\left(\varepsilon_{i_{1}}, \ldots, \varepsilon_{i_{l}}\right) \in C\left[(a, A), \mathbb{R}^{+l}\right]$ such that $z_{i}\left(t_{i}\right)=$ $x_{0 i}$ and the inequalities

$$
\begin{gathered}
\operatorname{sgn}\left(B_{j}-t_{j}\right)\left[\left(f_{j}(t, x)-z_{j}^{\prime}(t)\right)-h_{j}\left(t,\left(x_{j}-z_{j}(t)\right)_{+}\right)\right] \geq 0 \quad(j \in I) \\
\operatorname{sgn}\left(B_{j}-t_{j}\right)\left[-\left(f_{j}(t, x)-z_{j}^{\prime}(t)\right)-q_{j}\left(t,\left(x_{j}-z_{j}(t)\right)_{-}\right)\right] \geq 0 \quad(j \in I)
\end{gathered}
$$

are fulfilled on $\hat{\Omega}=\left\{(t, x): \varphi_{j}(t)<x_{j}-z_{j}(t)<\varphi_{j}(t)+\varepsilon_{j}(t), t \in \Delta\left(t_{j}, B_{j}\right)\right\}$ and $\hat{\hat{\Omega}}=$ $\left\{(t, x): \psi_{j}(t)<z_{j}(t)-x_{j}<\psi_{j}(t)+\varepsilon_{j}(t), t \in \Delta\left(t_{j}, B_{j}\right)\right\}$, respectively, for almost all $t \in \Delta\left(t_{j}, B_{j}\right)$. Then the Cauchy-Nicoletti boundary value problem (2.1) has at least two different solutions on $[a, A]$.

Proof. Without loss of generality, it can again be assumed that $I=\{1, \ldots, l\}$ and $\operatorname{Pr} x=$ $\left(x_{1}, \ldots, x_{l}\right)$. Put $k_{i}=2, g_{i 1}(t, u)=h_{i}(t, u), g_{i 2}(t, v)=q_{i}(t, v), \varphi_{i 1}(t)=\varphi_{i}(t), \varphi_{i 2}(t)=\psi_{i}(t)$, $V_{i 1}(t, x)=\left(x_{i}-z_{i}(t)\right)_{+}, V_{i 2}(t, x)=\left(x_{i}-z_{i}(t)\right)_{-}$, and $V_{i}(t, x)=\left(V_{i 1}(t, x), V_{i 2}(t, x)\right)$ for $i \in$ I. Then we have

$$
\begin{aligned}
\operatorname{sgn}\left(B_{i}-t_{i}\right) V_{i 1}^{\prime}(t, x(t)) & \geq \operatorname{sgn}\left(B_{i}-t_{i}\right)\left(f_{i}(t, x(t))-z_{i}^{\prime}(t)\right) \\
& \geq \operatorname{sgn}\left(B_{i}-t_{i}\right) g_{i 1}\left(t, V_{i 1}(t, x(t))\right), \\
\operatorname{sgn}\left(B_{i}-t_{i}\right) V_{i 2}^{\prime}(t, x(t)) & \geq-\operatorname{sgn}\left(B_{i}-t_{i}\right)\left(f_{i}(t, x(t))-z_{i}^{\prime}(t)\right) \\
& \geq \operatorname{sgn}\left(B_{i}-t_{i}\right) g_{i 2}\left(t, V_{i 2}(t, x(t))\right)
\end{aligned}
$$

for any solution $x=x(t)$ of $(2.1)$ a.e. on any interval $\left(\alpha_{i 1}, \alpha_{i 2}\right) \subseteq \Delta\left(t_{i}, B_{i}\right)$ for which

$$
V_{i 1}(t, x(t))<\varphi_{i}(t)+\varepsilon_{i}(t), \quad V_{i 2}(t, x(t))<\psi_{i}(t)+\varepsilon_{i}(t)
$$


on $\left(\alpha_{i 1}, \alpha_{i 2}\right), i=1, \ldots, l$, and

$$
V_{i 1}(t, x(t))>\varphi_{i}(t) \quad \text { or } \quad V_{i 2}(t, x(t))>\psi_{i}(t)
$$

on $\left(\alpha_{i 1}, \alpha_{i 2}\right)$, respectively. The statement follows from Theorem 2.1.

Corollary 2.7. Let the assumptions of Corollary 2.4 be fulfilled with the exception that conditions (i), (ii) are replaced by the following:

(i') there exist functions $h \in K_{a, A, t_{n}, B}\left[\hat{R}_{a, A}^{1}, \mathbb{R}\right]$ and $q \in K_{a, A, t_{n}, B}\left[\hat{R}_{a, A}^{1}, \mathbb{R}\right]$ such that $\operatorname{sgn}(t-$ $\left.t_{n}\right) h(t, \cdot)$ and $\operatorname{sgn}\left(t-t_{n}\right) q(t, \cdot)$ are nondecreasing and there are solutions $\varphi(t), \psi(t)$ of $u^{\prime}=h(t, u)$ and $v^{\prime}=q(t, v)$, respectively, satisfying

$$
\begin{gathered}
\varphi(t)>0, \quad \psi(t)>0 \quad \text { for } t \in \Delta\left(t_{n}, B\right), \quad \lim _{t \rightarrow t_{n}} \varphi(t)=\lim _{t \rightarrow t_{n}} \psi(t)=0, \\
\liminf _{t \rightarrow B} \varphi(t)>0, \quad \liminf _{t \rightarrow B} \psi(t)>0 ;
\end{gathered}
$$

(ii') there are $z \in \widetilde{A C}[[a, A], \mathbb{R}]$ and $\varepsilon \in C\left[(a, A), \mathbb{R}^{+}\right]$such that $z\left(t_{n}\right)=x_{0 n}$ and

$$
\begin{gathered}
\operatorname{sgn}\left(B-t_{n}\right)\left[\tilde{f}\left(t, x_{1}, \ldots, x_{n}\right)-z^{\prime}(t)-h\left(t,\left(x_{n}-z(t)\right)_{+}\right)\right] \geq 0, \\
\operatorname{sgn}\left(B-t_{n}\right)\left[-\tilde{f}\left(t, x_{1}, \ldots, x_{n}\right)+z^{\prime}(t)-q\left(t,\left(x_{n}-z(t)\right)_{-}\right)\right] \geq 0
\end{gathered}
$$

hold on $\hat{\Omega}=\left\{\left(t, x_{1}, \ldots, x_{n}\right): \varphi(t)<x_{n}-z(t)<\varphi(t)+\varepsilon(t), t \in \Delta\left(t_{n}, B\right)\right\}$ and $\hat{\hat{\Omega}}=$ $\left\{\left(t, x_{1}, \ldots, x_{n}\right): \psi(t)<z(t)-x_{n}<\psi(t)+\varepsilon(t), t \in \Delta\left(t_{n}, B\right)\right\}$, respectively, for almost all $t \in \Delta\left(t_{n}, B\right)$. Then the Cauchy-Nicoletti boundary value problem (2.37) has at least two different solutions on $[a, A]$.

Proof. Corollary 2.7 follows from Corollary 2.6 in the same way as Corollary 2.4 follows from Corollary 2.2.

Example 2.8. Let $p_{1} \in K_{1}\left[\hat{R}_{0,1}^{2}, \mathbb{R}\right]$ and $p_{2} \in K_{1}\left[\hat{R}_{0,1}^{2}, \mathbb{R}\right]$ be such that

$$
\begin{array}{cl}
p_{1}\left(t, x_{1}, x_{2}\right) \leq-\delta_{1}(t) \vartheta_{1}\left(x_{2}\right) & \text { for }\left(t, x_{1}, x_{2}\right) \in(0,1) \times \mathbb{R} \times(0, \infty), \\
p_{1}\left(t, x_{1}, x_{2}\right) \geq \delta_{2}(t) \vartheta_{2}\left(\left|x_{2}\right|\right) & \text { for }\left(t, x_{1}, x_{2}\right) \in(0,1) \times \mathbb{R} \times(-\infty, 0), \\
x_{2} p_{2}\left(t, x_{1}, x_{2}\right) \leq 0 & \text { for }\left(t, x_{1}, x_{2}\right) \in(0,1) \times \mathbb{R}^{2},
\end{array}
$$

where $\delta_{1}, \delta_{2}$ are positive functions such that $\delta_{j} \in L[[0,1], \mathbb{R}]$ and $\vartheta_{j} \in C\left[[0, \infty), \mathbb{R}^{+}\right](j=$ $1,2)$ are nondecreasing and positive on $(0, \infty)$ and satisfying $\vartheta_{1}(0)=\vartheta_{2}(0)=0, \int_{0}^{1} \delta_{1}(s) d s<$ $\int_{0}^{\infty} 1 / \vartheta_{1}(s) d s<\infty$, and $\int_{0}^{1} \delta_{2}(s) d s<\int_{0}^{\infty} 1 / \vartheta_{2}(s) d s<\infty$.

Consider the boundary value problem

$$
w^{\prime \prime}=p_{1}\left(t, w, w^{\prime}\right)+p_{2}\left(t, w, w^{\prime}\right), \quad w(0)=0, \quad w^{\prime}(1)=0 .
$$


It holds that

$$
\begin{gathered}
-\left[p_{1}\left(t, x_{1}, x_{2}\right)+p_{2}\left(t, x_{1}, x_{2}\right)+\delta_{1}(t) \vartheta_{1}\left(x_{2}\right)\right] \geq 0 \\
\text { for }\left(t, x_{1}, x_{2}\right) \in(0,1) \times \mathbb{R} \times(0, \infty), \\
-\left[-p_{1}\left(t, x_{1}, x_{2}\right)-p_{2}\left(t, x_{1}, x_{2}\right)+\delta_{2}(t) \vartheta_{2}\left(-x_{2}\right)\right] \geq 0 \\
\text { for }\left(t, x_{1}, x_{2}\right) \in(0,1) \times \mathbb{R} \times(-\infty, 0) .
\end{gathered}
$$

The problems

$$
\begin{aligned}
u^{\prime}=-\delta_{1}(t) \vartheta_{1}(u), & u(1)=0, \\
v^{\prime}=-\delta_{2}(t) \vartheta_{2}(v), & v(1)=0
\end{aligned}
$$

have positive solutions on $[0,1)$ and condition (2.54) implies

$$
\left[p_{1}\left(t, x_{1}, x_{2}\right)+p_{2}\left(t, x_{1}, x_{2}\right)\right] \operatorname{sgn} x_{2} \leq 0 .
$$

Therefore, the assumptions of Corollary 2.7 are fulfilled with $a=0, A=1, c=0, z(t) \equiv 0$, $B=0, t_{1}=0, t_{2}=1, \lambda(t) \equiv 0, \mu(t) \equiv 0$, and

$$
\begin{aligned}
& h(t, u)= \begin{cases}-\delta_{1}(t) \vartheta_{1}(u) & \text { for }(t, u) \in(0,1) \times(0, \infty), \\
0 & \text { for }(t, u) \in(0,1) \times(-\infty, 0],\end{cases} \\
& q(t, v)= \begin{cases}-\delta_{2}(t) \vartheta_{2}(v) & \text { for }(t, v) \in(0,1) \times(0, \infty), \\
0 & \text { for }(t, v) \in(0,1) \times(-\infty, 0] .\end{cases}
\end{aligned}
$$

Hence problem (2.53) has at least two solutions on $[0,1]$.

\section{Acknowledgment}

The research was supported by the Council of Czech Government (J 07/98: 143100001).

\section{References}

[1] R. P. Agarwal and V. Lakshmikantham, Uniqueness and Nonuniqueness Criteria for Ordinary Differential Equations, Series in Real Analysis, vol. 6, World Scientific Publishing, New Jersey, 1993.

[2] J. Andres, Ordinary differential equations in the lack of uniqueness, Atti Sem. Mat. Fis. Univ. Modena 49 (2001), no. 1, 247-267.

[3] J. Kalas, Nonuniqueness for the solutions of ordinary differential equations, Czechoslovak Math. J. 29 (1979), no. 1, 105-112.

[4]_, General nonuniqueness theorem for ordinary differential equations, Dynam. Contin. Discrete Impuls. Systems 3 (1997), no. 1, 97-111.

[5] _ Nonuniqueness results for ordinary differential equations, Czechoslovak Math. J. 48 (1998), no. 2, 373-384.

[6] Nonuniqueness theorem for a singular Cauchy problem, Georgian Math. J. 7 (2000), no. 2, 317-327.

[7] I. T. Kiguradze, The comparison lemma and the question of uniqueness for solutions of the Cauchy problem for ordinary differential equations, Soobšč. Akad. Nauk Gruzin. SSR 39 (1965), 513518 (Russian). 


\section{Nonuniqueness for a singular Cauchy-Nicoletti problem}

[8] - On the Cauchy problem for ordinary differential equations with a singularity, Soobšč. Akad. Nauk Gruzin. SSR 37 (1965), 19-24 (Russian).

[9] On the Cauchy problem for singular systems of ordinary differential equations, Differ. Uravn. 1 (1965), 1271-1291 (Russian).

[10] - On a singular problem of Cauchy-Nicoletti, Ann. Mat. Pura Appl. (4) 104 (1975), 151175.

[11] On the modified problem of Cauchy-Nicoletti, Ann. Mat. Pura Appl. (4) 104 (1975), $177-186$.

[12] Some Singular Boundary Value Problems for Ordinary Differential Equations, Izdat. Tbilis. Univ., Tbilisi, 1975.

[13] V. Lakshmikantham, On the Kamke's function in the uniqueness theorem of ordinary differential equations, Proc. Nat. Acad. Sci. India Sect. A 34 (1964), 11-14.

[14] C. Nowak, Some remarks on a paper by M. Samimi on: "Nonuniqueness criteria for ordinary differential equations", Appl. Anal. 47 (1992), no. 1, 39-44.

[15] C. Nowak and H. Stettner, Nonuniqueness results for ordinary differential equations, Nonlinear Anal. 30 (1997), no. 6, 3935-3938.

[16] M. Samimi, Nonuniqueness criteria for ordinary differential equations, Applicable Anal. 13 (1982), no. 4, 291-296.

[17] H. Stettner, Nichteindeutigkeit bei gewöhnlichen differentialgleichungen, Math. Nachr. 64 (1974), 233-237 (German).

Josef Kalas: Department of Mathematical Analysis, Masaryk University Brno, Janáčkovo nám. 2a, 66295 Brno, Czech Republic

E-mail address: kalas@math.muni.cz 


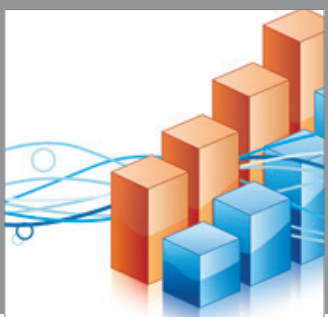

Advances in

Operations Research

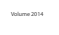

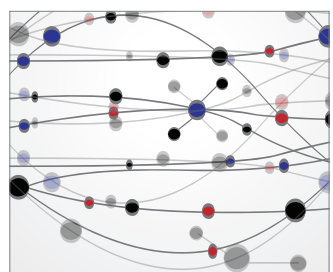

\section{The Scientific} World Journal
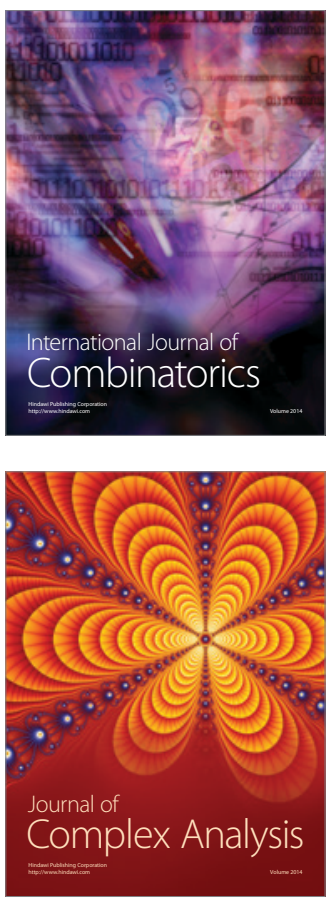

International Journal of

Mathematics and

Mathematical

Sciences
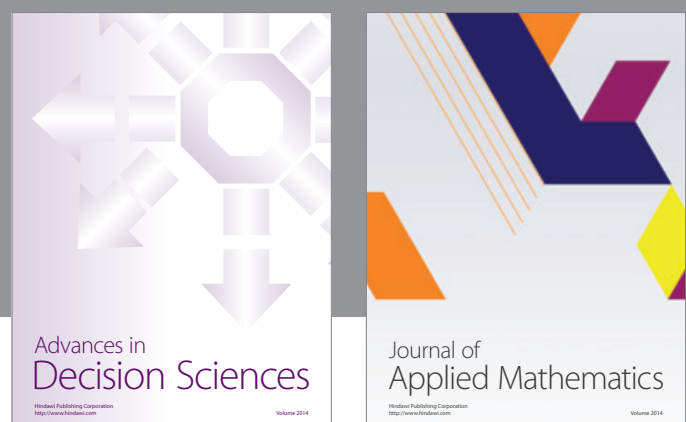

Journal of

Applied Mathematics
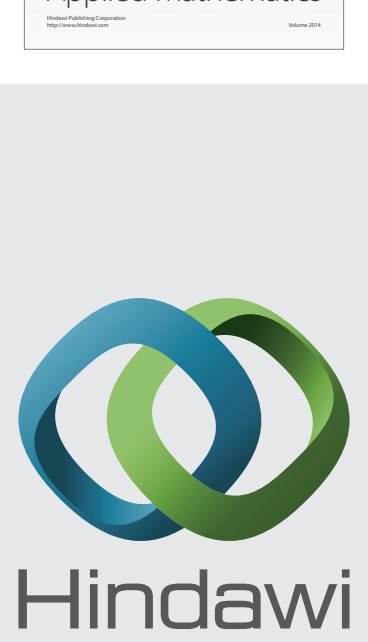

Submit your manuscripts at http://www.hindawi.com
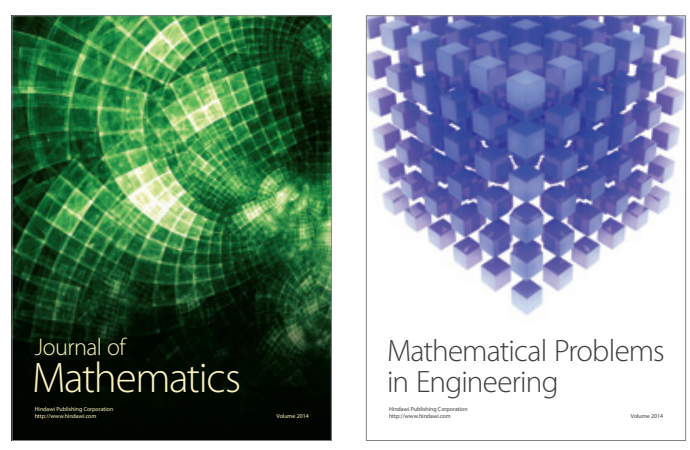

Mathematical Problems in Engineering
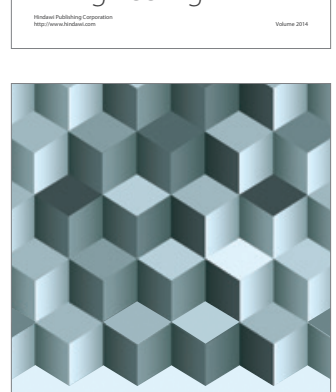

Journal of

Function Spaces
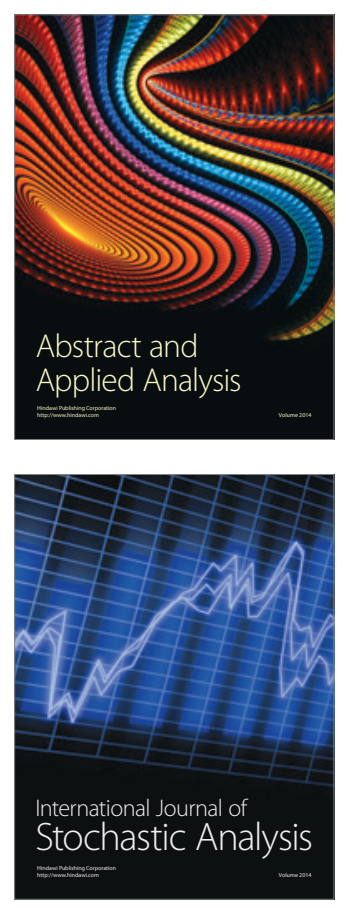

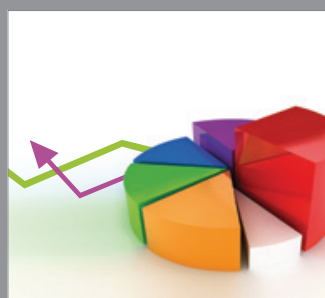

ournal of

Probability and Statistics

Promensencen
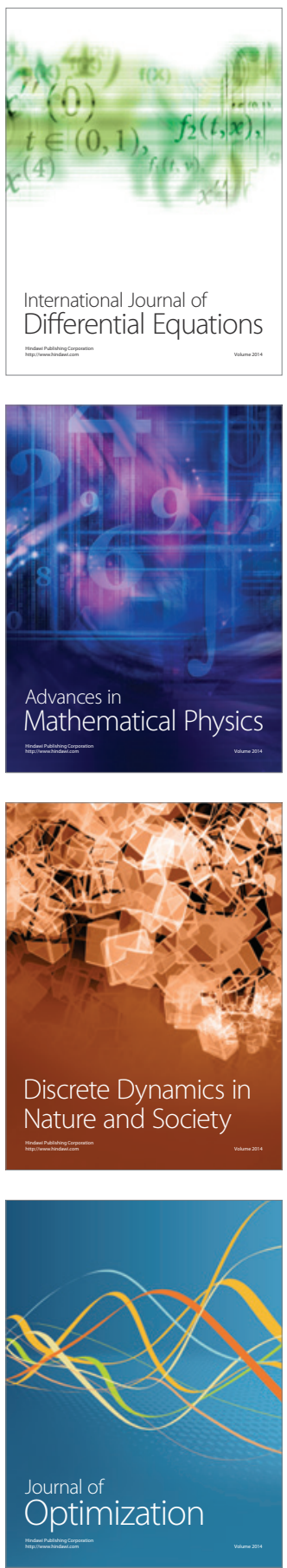